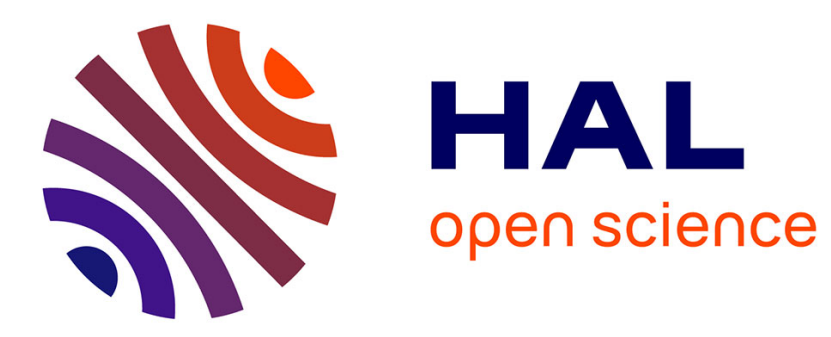

\title{
La Cour de Justice et le contrôle démocratique de l'Union européenne
}

\author{
Olivier Costa
}

\section{To cite this version:}

Olivier Costa. La Cour de Justice et le contrôle démocratique de l'Union européenne. Revue Française de Science Politique, 2001, 51 (6), pp.881-902. 10.3406/rfsp.2001.403683 . halshs-00259281

\section{HAL Id: halshs-00259281 https://shs.hal.science/halshs-00259281}

Submitted on 9 Apr 2019

HAL is a multi-disciplinary open access archive for the deposit and dissemination of scientific research documents, whether they are published or not. The documents may come from teaching and research institutions in France or abroad, or from public or private research centers.
L'archive ouverte pluridisciplinaire HAL, est destinée au dépôt et à la diffusion de documents scientifiques de niveau recherche, publiés ou non, émanant des établissements d'enseignement et de recherche français ou étrangers, des laboratoires publics ou privés.

\section{(1) (1) $\$$}

Distributed under a Creative Commons Attribution - NonCommercial - NoDerivatives| 4.0 


\section{La Cour de justice et le contrôle démocratique de l'Union} européenne

Monsieur Olivier Costa

\section{Citer ce document / Cite this document :}

Costa Olivier. La Cour de justice et le contrôle démocratique de l'Union européenne. In: Revue française de science politique, $51^{\mathrm{e}}$ année, $\mathrm{n}^{\circ} 6,2001$. pp. 881-902;

https://www.persee.fr/doc/rfsp_0035-2950_2001_num_51_6_403683

Fichier pdf généré le 26/04/2018 


\title{
Résumé
}

Face aux difficultés que pose la légitimation du système politique de l'Union européenne, les juristes insistent sur la contribution spécifique de la Cour de justice à ce processus. En reconnais-sant aux citoyens de nombreux droits et en leur permettant de les faire sanctionner par divers biais auprès d'elle, la Cour les ferait accéder à un certain degré de contrôle sur les décisions des ins-titutions communautaires. Si l'on se penche sur la jurisprudence et la pratique de la Cour, on constate cependant que les voies de recours sont étroites et qu'il existe de grandes asymétries quant à l'accès des particuliers au prétoire communautaire, que ce soit en fonction de leur natio-nalité ou de leur degré d'expertise sur les questions européennes. Les juges communautaires sem-blent d'ailleurs peu pressés de remédier à cette situation. Défait, il est douteux que les possibilités de recours des citoyens devant la Cour contribuent significativement à les rapprocher des acteurs de l'Union et, partant, à améliorer la légitimation de celle-ci. Aussi, la « démocratie judiciaire » à l'échelle supranationale consiste-t-elle pour l'heure en une simple judiciarisation du politique.

\begin{abstract}
The european court of justice and democratic control of the european union Legal scholars insist on the specific contribution of the European Court of Justice to the legitimation of the Union's political system. By recognizing many rights to citizens and by making it possible to uphold them by various means, the Court is seen as enabling them to exercise a certain degree of control over the Union institutions'decisions. If one looks at the case-law and the practices of the Court, however, the means of recourse are limited, and there are many disparities in individuals' access to the Union's courtroom, which depends on nationality and the degree of knowledge of European questions. The Union's judges do not seem in a great hurry to remedy this situation. It is dubious that the possibilities of citizens to appeal to the Court will contribute significantly to brin-ging them closer to the Union's actors, and thereby improve its legitimacy. "Judicial democracy » on the supranational level thus seems to consist of a mere judicialization of politics.
\end{abstract}




\title{
LA COUR DE JUSTICE \\ ET LE CONTRÔLE DÉMOCRATIQUE DE L'UNION EUROPÉENNE
}

\author{
OLIVIER COSTA
}

$\mathrm{E}$

n créant les Communautés européennes, les «Pères fondateurs » ont mis en place un système institutionnel hybride, reposant sur l'intrication étroite de plusieurs logiques de fonctionnement. Le droit devait assurer la cohérence et l'efficacité de l'ensemble, être à la fois le moteur de l'intégration européenne, le langage commun de ses acteurs et le régulateur des rapports interinstitutionnels. Aussi, la Cour de justice des Communautés européennes (CJCE), qui était chargée d'interpréter le droit communautaire et de veiller à sa bonne application, a-t-elle joué d'emblée un rôle clé et suscité, dès les années 1960, deux discours contradictoires. Le premier, émanant des responsables politiques et des analystes les plus critiques à l'égard du processus d'intégration supranational et de ses modalités, dénonçait les dérives de la jurisprudence de la Cour et la manière très libre dont ses juges interprétaient les traités pour servir l'objectif de construction européenne, assurer l'indépendance de ses institutions et donner sa force au đroit communautaire. Les juges étaient accusés de promouvoir ouvertement l'intégration européenne sans tenir compte de la permanence des États-nations et de l'avis des principaux intéressés qu'étaient les citoyens. Un second discours, tenu par les acteurs et les spectateurs les plus enthousiastes de l'intégration européenne, prit rapidement le contre-pied de cette dénonciation du « gouvernement des juges communautaires ». Il soulignait pour sa part la contribution de la Cour au respect par les institutions européennes et les acteurs économiques de leurs obligations, et par les États-membres de leurs engagements, délibérément souscrits lors de la ratification des traités. Il portait également au crédit de la Cour la « démocratisation » du système institutionnel de l'Union - notamment par le renforcement des prérogatives et de l'influence du Parlement européen - et la reconnaissance aux citoyens européens de nombreux droits.

Cette controverse autour des vices et vertus de la Cour - à laquelle un Tribunal de première instance a été adjoint en $1988^{`}$ - $s^{\prime}$ est quelque peu tempérée ces dernières années. Les principaux apports de sa jurisprudence sont d'autant moins contestés que la Cour n'a plus été amenée à rendre des arrêts aussi spectaculaires que par le passé. De fait, les débats, réflexions et rapports sur l'avenir de l'Union et la réforme de ses institutions qui se sont multipliés depuis la fin des années 1990 n'ont guère abordé la question d'une réforme des compétences ou du mode de fonctionnement de la Cour. Au contraire, celle-ci est aujourd'hui perçue comme une ressource et un point d'appui pour accomplir de nouveaux progrès sur la voie de l'intégration européenne ou, à

1. Le Conseil a créé par une décision du 24 octobre 1988 un Tribunal de première instance, destiné à faire face à l'engorgement de la Cour. Il est, entre autres, compétent pour examiner les recours directs des particuliers, ces derniers pouvant faire appel des décisions du Tribunal devant la Cour. Au sens des traités et d'un point de vue budgétaire, le Tribunal ne constitue pas une institution distincte de la Cour. Aussi, le terme «Cour »sera-t-il employé ici de manière générique. Cf., entre autres, J.-C. Gautron, « Les compétences du Tribunal de première instance des Communautés européennes », Revue du Marché commun et de l'Union européenne, 1995, p. 568-575. 
l'inverse, pour en limiter les excès. Quoi qu'on en pense, la Cour de justice est l'une des clés du fonctionnement de la Communauté ${ }^{1}$, non seulement pour garantir le respect de son droit, mais aussi pour assurer la limitation mutuelle des activités de ses acteurs, qu'il s'agisse des institutions de l'Union, des États-membres ou des particuliers. La Cour n'a pas l'exclusivité de l'examen des conflits qui opposent ces acteurs, mais elle tranche souvent en dernier ressort et contribue, ce faisant, au contrôle qu'ils exercent les uns sur les autres.

En réponse aux contempteurs du « déficit démocratique européen », les avocats de l'intégration européenne ont ainsi invoqué l'existence de diverses voies permettant à toute «personne physique ou morale » de demander à la Cour d'interpréter ou de contrôler la légalité d'une règle de droit communautaire, voire de contester certaines décisions des institutions de la Communauté. La Cour de justice a en effet affirmé très tôt le caractère fédéral du système juridictionnel communautaire et insisté sur ses implications pour les citoyens européens. Elle a, entre autres, établi qu'ils étaient directement et individuellement concemés par les normes communautaires et qu'ils pouvaient s'en prévaloir. Dès 1963, dans leur arrêt Van Gend en Loos ${ }^{2}$, les juges ont estimé que la « vigilance des particuliers intéressés à la sauvegarde de leurs droits » confortait le contrôle exercé par les institutions communautaires sur l'application par les États du droit communautaire. Is ont ainsi choisi de lier les objectifs de protection des droits individuels et de bonne application du droit communautaire dans les ordres juridiques nationaux. Les juges, de même que la plupart des spécialistes de droit communautaire, ont tiré argument des possibilités d'accès des citoyens à la Cour et des audaces de sa jurisprudence pour souligner sa contribution spécifique, non seulement à l'intégration européenne, mais aussi à l'identification des implications positives de ce processus pour les citoyens ${ }^{3}$. Selon ces acteurs et auteurs, la Cour contribuerait à pallier, à sa mesure, le déficit démocratique et l'élitisme qui affectent le système politique européen, en veillant aux équilibres institutionnels, en préservant les droits fondamentaux des citoyens et en leur ménageant la possibilité de contester les décisions communautaires qui leur font directement grief.

Les nombreux travaux juridiques qui ont été consacrés à ce sujet sont focalisés sur les modalités de l'accès des citoyens aux voies de recours, sur le contenu des arrêts et sur la logique d'ensemble du système judiciaire, et ne proposent pas d'analyse quantitative ou qualitative des auteurs de recours directs ${ }^{4}$. Aussi, nous semble-t-il utile de saisir cette question avec les outils de la science politique et d'aller au-delà du raisonnement des juges et de la lettre des traités. Comme dans toute analyse du système politique de l'Union européenne ${ }^{5}$, il importe de ne pas distinguer à l'excès droit et poli-

1. Pour une analyse de la contribution de la Cour à l'intégration européenne, cf. R. Dehousse, The European Court of Justice : The Politics of Judicial Integration, New York, St. Martin's Press, 1998 ; A. Stone Sweet, J. A. Caporaso, « La Cour de justice et l'intégration européenne », Revue française de science politique, 48 (2), avril 1998, p. 195-243.

2. Arrêt de la Cour, Van Gend en Loos, aff. 26/62, 1963, Rec. p. 1.

3. Le juge G. F. Mancini était l'un des principaux promoteurs de cette vision des choses. Cf. «Democracy and the European Court of Justice », Modern Law Review, 57, 1994, p. 186 et suiv. (avec D. T. Keeling) ; G. F. Mancini (dir.), Democracy and Constitutionalism in the European Union: Collected Essays, Oxford, Hart Publishers, 2000.

4. À l'exception notable de C. Harding : "Who Goes to Court in Europe? An Analysis of Litigation against the European Community », European Law Review, 17 (2), 1992, p. 105125.

5. Cf. G. Duprat (dir.), L'Union européenne, droit, politique, démocratie, Paris, PUF, 1996. Cf. également les contributions à la journée d'étude du groupe «Europe » de l'Association française de science politique : «Droit et politique dans I'Union européenne», Paris, 16 mars 2001 (<www.afsp.msh-paris.fr>). 
tique, et d'envisager le droit comme un instrument de fins politiques ou économiques. Rappelons que le processus d'intégration européenne repose dans son ensemble sur cette logique juridique instrumentale : elle permet de rendre compte des relations interinstitutionnelles, mais aussi de comprendre les interactions du système politique avec son environnement. C'est pourquoi, il nous semble que suivre sans prévention le raisonnement de la Cour et considérer la Communauté européenne comme un système de droit complet et cohérent protégeant les droits et intérêts des individus, revient à postuler une proximité entre le système politique européen et les citoyens dont les manifestations ne sont pas frappantes. On entend donc évaluer les possibilités concrètes d'accès des particuliers au prétoire de la Cour de justice. Plus précisément, il s'agit d'identifier les auteurs de recours et de cerner, au-delà du discours de principe, l'attitude des juges européens face à ces interventions. Ce faisant, on pourra appréhender la contribution de la Cour de justice à la défense des intérêts des particuliers et à leur capacité de contrôle sur le policy-making de l'Union.

\section{LES PARTICULIERS ET LE SYSTÈME JUDICIAIRE DES COMMUNAUTÉS : DISCOURS ET DISPOSITIFS}

\section{L'OUVERTURE DU PRÉTOIRE COMMUNAUTAIRE AUX CITOYENS ET LA LÉGITIMATION DE L'UNION}

L'Union européenne entretient peu de contacts avec les citoyens : sa légitimation est, aujourd'hui encore, essentiellement procédurale et intrasystémique. Elle ne peut guère compter sur une légitimation substantielle ; les politiques communautaires, qui sont faiblement redistributives à l'égard des particuliers et manquent de visibilité, ne permettent pas de susciter l'adhésion des citoyens européens sur un mode clientéliste '. Les tentatives de légitimer le système par la participation n'ont pas davantage porté leurs fruits ; les élections européennes, qui constituent le principal vecteur de cette participation, se singularisent par un taux d'abstention croissant et par la focalisation répétée des campagnes sur des thématiques nationales. Les institutions européennes ont cherché à diversifier les canaux de la participation, en multipliant les

1. Les travaux et actions menés grâce aux fonds structurels communautaires jouissent d'une large publicité et peuvent susciter un certain soutien au projet d'intégration européenne. Les sondages Eurobaromètre révèlent ainsi une plus forte adhêsion des citoyens à l'Union européenne dans les régions qui bénéficient de ses politiques de redistribution; de même, le taux de participation aux élections européennes y est supérieur à la moyenne. Il faut toutefois se défier d'une vision caricaturale du «citoyen-consommateur » et noter que ce soutien est très volatile et s'adresse davantage à un secteur d'action de l'Union qu'au système politique dans son ensemble. Dans un autre contexte, David Easton distinguait ainsi le soutien diffus que les citoyens apportent à un gouvernement - qui relève de leur adhésion au système politique -, de leur soutien spécifique - qui répond à une politique ou un avantage (« $\mathrm{A} R \mathrm{Re}$-Assessment of the Concept of Political Support », British Journal of Political Science, 5, 1975, p. 435-457). L'échec du référendum sur la ratification du traité de Nice en Irlande, le 8 juin 2001, n'est certainement pas étranger à ce phénomène de différenciation entre, d'une part, l'attachement que les citoyens portent à certaines politiques de l'Union (notamment à sa politique structurelle) et, d'autre part, celui qu'ils manifestent à l'endroit du processus d'intégration européenne luimême. 
consultations et en suscitant un «dialogue civil européen », mais cela ne concerne guère le citoyen lambda. Pour reprendre le schéma systémiste de David Easton ${ }^{1}$, l'Union européenne se présente comme un système politique qui peine à nouer des relations avec ses citoyens, aussi bien par les « outputs» (politiques publiques, redistribution) que par les « inputs » (participation, consultations, « opinion publique »).

Cette situation a poussé les acteurs de l'Union et les zélateurs de l'intégration européenne à préconiser la diversification des procédures de contrôle internes au système, et les incite à souligner la participation des individus à ce contrôle par le biais de la « citoyenneté judiciaire » que leur a reconnu la Cour ${ }^{2}$. Il est vrai que les arrêts de celle-ci sont de plus en plus médiatisés et ont un impact grandissant sur la vie quotidienne des citoyens et des acteurs économiques et sociaux européens. Nous en avons eu de multiples exemples ces dernières années : l'arrêt Bosman (1995) est venu bouleverser le monde du sport d'équipe ; la condamnation du constructeur automobile Volkswagen (2000) a réaffirmé avec force le principe de libre circulation des produits ; l'annulation de la directive sur la publicité pour le tabac (2000) a, quant à elle, illustré la contribution de la Cour au respect des prérogatives des États et du principe de subsidiarité. Toutefois, si la conscience se fait chez les citoyens que la juridiction communautaire joue un rôle important dans l'Union, on ne sait quelle est leur perception des possibilités d'action auprès d'elle. En outre, qu'en est-il du « droit au juge » que les membres de la Cour présentent comme une priorité de leur action?

Dans leur discours public, les juges communautaires soulignent à l'envi la place que les citoyens occupent dans leurs activités ${ }^{3}$. Ils considèrent - à l'image de leurs homologues de la Cour européenne des droits de l'homme - que le principe du « droit au juge » est un élément central de l'État de droit, voire un droit fondamental du citoyen européen. La Cour de justice a affirmé que les États-membres devaient mettre en place, pour assurer la réalité des droits fondés sur le droit communautaire, des procédures qui ne soient pas moins favorables que celles visant à la sauvegarde des droits fondés sur les dispositions nationales (principe de « traitement égal ») et que ces procédures devaient être réellement utilisables (principe « d'effectivité ») ${ }^{4}$. La protection juridictionnelle des individus est très large, puisqu'elle s'étend à l'ensemble des droits dérivés du droit communautaire, et non pas aux seuls « droits civils», comme c'est le cas dans la jurisprudence de la Cour européenne des droits de l'homme. Le ton est le même dans la brochure « vos questions sur la Cour de justice des Communautés européennes ${ }^{5}$, que l'institution destine au grand public. La contribution de la Cour au bien-être et à la défense des droits des citoyens est envisagée sous tous les angles, et les rédacteurs laissent entendre que là réside la mission première de la juridiction

1. D. Easton, Analyse du système politique, Paris, Armand Colin, 1974 (1 irc éd. : 1965). Pour une application à l'Union européenne, cf. F. Scharpf, Gouverner l'Europe, Paris, Presses de Sciences Po, 2000.

2. Cette situation a également incité les négociateurs du traité de Maastricht à introduire dans l'article relatif à la citoyenneté européenne la formule suivante : «Les citoyens de l'Union jouissent des droits et sont soumis aux devoirs prévus par le présent traité » (article $17 \S 2 \mathrm{du}$ traité CE). Cf. P. Magnette, La citoyenneté européenne. Droits, politiques, institutions, Bruxelles, Éditions de l'Université de Bruxelles, 1999.

3. À l'occasion d'une journée d'étude organisée le 19 octobre 1999 pour célébrer les dix années d'activité juridictionnelle du Tribunal de première instance, un des thèmes de réflexion fut ainsi consacré à la protection juridictionnelle des justiciables.

4. Sur ce point, cf. J. Dutheil de la Rochère, «Droit au juge, accès à la justice européenne », Pouvoirs, 96, janvier 2001, p. 128-132.

5. CJCE, «Vos questions sur la Cour de justice des Communautés européennes », Luxembourg, 1999, réf. DX-23-99-823-FR-D. 
communautaire. Cette courte brochure affirme que «le droit communautaire concerne les citoyens au plus près de leurs diverses activités » et que « la Cour de justice, en interprétant ce droit et en le faisant respecter, est donc amenée à prendre en compte les préoccupations des citoyens dans différents aspects de leur vie quotidienne ». Elle précise que « le citoyen a la possibilité d'accéder à la Cour ou au Tribunal par le biais de deux mécanismes ", l'un indirect (le renvoi préjudiciel), l'autre direct (le recours direct). Ce document souligne enfin les «facilités » qui sont offertes aux citoyens : aide judiciaire, gratuité des recours, possibilité de s'adresser à la Cour dans n'importe laquelle des langues officielles de l'Union.

\section{DES VOIES DE RECOURS COMPLEXES ET ÉTROITES}

L'examen des possibilités concrètes d'intervention des particuliers devant la Cour de justice et le Tribunal de première instance révèle de fortes distorsions, selon qu'il s'agit de personnes morales ou physiques, et selon leurs moyens et leur connaissance du système. Que l'on se penche sur les recours directs (recours en annulation pour l'essentiel, mais aussi exception d'illégalité, recours en carence, appel contre des sanctions spécifiques, action en réparation de dommages, contestation d'aides d'État...) ou sur les renvois préjudiciels, l'étude de la jurisprudence et de l'identité des plaignants montre que ces procédures ne profitent qu'à des catégories limitées d'acteurs. Les avocats des cabinets spécialisés en droit communautaire, qui introduisent une large partie des recours directs à Luxembourg, affirment être essentiellement sollicités par des multinationales et des grands groupes d'intérêt européens. Les juges et les référendaires qui les assistent l'admettent, mais font valoir que de nombreux recours sont également introduits sans l'aide de ces cabinets par de petites entreprises, des agriculteurs ou des indépendants. Précisons un peu.

Si la Cour a élaboré sa jurisprudence avec le souci de mettre en place un ordre juridique complet régi par la règle de droit ', ses efforts se sont révélés plus mesurés en direction des plaignants à titre individuel que des institutions. Le traité CEE prévoyait, dès 1957, la possibilité d'un recours en annulation contre les actes des institutions investies du pouvoir de décision, inspiré par le recours pour excès de pouvoir français. Toutefois, il établissait une hiérarchie très nette entre les plaignants. Aujourd'hui encore, l'article 230 du traité CE prévoit que les États-membres, le Conseil et la Commission appartiennent à une catégorie de plaignants privilégiés qui peuvent demander sans condition l'annulation d'un acte ; la Banque centrale européenne, la Cour des Comptes et le Parlement européen se situent sur un second rang, et ne peuvent agir que pour défendre leurs prérogatives ${ }^{2}$; enfin, il est prévu que « toute personne physique ou morale peut former ... un recours contre les décisions dont elle est le destinataire et contre les décisions qui, bien que prises sous l'apparence d'un règlement ou d'une décision adressée à une autre personne, la concernent directement et individuellement ».

1. De nombreux ouvrages relatifs à la jurisprudence de la Cour de justice précisent ce point. Cf., entre autres, A. Arnull, The European Union and its Court of Justice, New York, Oxford University Press, $1999 ;$ K. P. E. Lasok, The European Court of Justice : Practice and Procedure, Londres, Butterworths, 1994 ( $2^{c}$ éd.) ; D. Simon, Le système juridique communautaire, Paris, PUF, 1999 ( $2^{c}$ éd.).

2. Lors de son entrée en vigueur, le traité de Nice modifiera l'article $230 \mathrm{CE}$ pour permettre au Parlement européen d'agir auprès de la Cour sans condition. 
Il n'est pas possible, dans le cadre de cet article, d'examiner en détail la jurisprudence complexe, fluctuante et délicate d'interprétation relative à cette question ${ }^{1}$. Retenons que les juges se sont essentiellement focalisés sur la détermination de la nature individuelle ou collective des actes contestés et ont établi une jurisprudence très restrictive sur ce point. Une libéralisation a eu lieu, mais elle est restée circonscrite à trois secteurs : la concurrence, les mesures antidumping et les aides d'État. Dans ces domaines, la Cour a reconnu que des mesures, même législatives, pouvaient affecter des individus de manière particulière et leur ouvrir de ce fait un droit de recours. L'arrêt Codorniu ${ }^{2}$ a établi en 1994 qu'il n'était plus nécessaire que le recours concerne une mesure individuelle. La Cour ne se prononça toutefois qu'à l'issue de vifs débats entre les juges partisans et adversaires d'une ouverture accrue des recours directs aux particuliers, et ce, dans un contexte inhabituel où les enjeux économiques n'étaient (selon les acteurs de la Cour que l'on a pu interroger à ce sujet) pas étrangers aux positions des uns et des autres. La jurisprudence de la Cour connut ainsi des évolutions sur ce point, au gré notamment de l'arrivée de nouveaux juges ${ }^{3}$. Le Tribunal de première instance, qui prit en charge l'examen des recours des particuliers quelque temps plus tard, se montra peu pressé de donner suite à l'arrêt Codorniu et revint souvent à la jurisprudence antérieure ${ }^{4}$. Cet arrêt, qui semblait annoncer le déclin de la tendance de la Cour à protéger systématiquement les institutions de l'Union contre les recours des particuliers ${ }^{5}, n^{\prime}$ a donc pas déclenché l'ouverture attendue. Les particuliers disposent certes d'autres possibilités d'action, mais elles sont d'un emploi beaucoup moins fréquent ${ }^{6}$. Aujourd'hui, malgré quelques évolutions, il existe donc un décalage persistant entre les droits que les particuliers possèdent en vertu de l'intégration européenne et ceux qu'ils peuvent faire sanctionner.

On ne peut pour autant dénier à l'Union le caractère de « communauté de droit ». Les individus ont en effet bénéficié dès 1963 d'une interprétation très libérale du prin-

1. Cf. P. Nihoul, «La recevabilité des recours en annulation introduits par un particulier à l'encontre d'un acte communautaire de portée générale $»$, Revue trimestrielle de droit européen, 1994 , p. 171 et suiv. Pour une synthèse actualisée et accessible aux non-juristes, on renverra à notre article à paraître dans la Revue internationale de politique comparée : « Les citoyens et le droit communautaire : les usages élitaires des voies de recours devant les juridictions de l'Union », 3, 2001.

2. Arrêt de la Cour, Codorniu SA v. Conseil, aff. 309/89, 1994, Rec. p. I-1853.

3. Avant la fin de l'année du jugement (1994), quatre juges quittèrent la Cour, tandis que trois juges issus des nouveaux États-membres prirent leurs fonctions au début de l'année suivante.

4. Ce constat est celui, concordant, de plusieurs référendaires et fonctionnaires de la Cour et du Tribunal. Certains auteurs sont toutefois d'un avis contraire. Georges Vandersanden écrit ainsi : « La jurisprudence du Tribunal montre que celui-ci, tout en étant respectueux de l'interprétation restrictive donnée par la Cour de justice à ces notions [intérêt direct et individuel], s'est efforcé, dans la mesure du possible, d'en assouplir la rigueur au profit de la recevabilité des actions des particuliers ». Il ajoute néanmoins : « Toutefois, cette jurisprudence, sans être incohérente, n'est pas toujours homogène et il peut être difficile de réconcilier certains arrêts, si ce n'est par une attention particulière portée sur les faits spécifiques et les procédures en cause ». "Le Tribunal de première instance des Communautés européennes: dix ans d'existence », dans P. Magnette, E. Remacle, Le nouveau modèle européen. Vol. 1. Institutions et gouvernance, Bruxelles, Éditions de l'Université de Bruxelles, 2000, p. 111-112.

5. J.-C. Carvalho Montinho de Almeida, «Le recours en annulation des particuliers (article 173, deuxième alinéa, du traité $\mathrm{CE}$ ): nouvelles réflexions sur l'expression "la concernent individuellement" », dans Festchrift für Ulrich Everling, Bd 1, 1995, p. 849 et suiv.

6. Il s'agit du recours en carence (art. $232 \mathrm{CE}$ ), de l'exception d'illégalité (art. $241 \mathrm{CE}$ ), de l'appel contre des sanctions prévues par certains règlements (art. $229 \mathrm{CE}$ ), de l'action en réparation de dommages (art. 235 et $288 \mathrm{CE}$ ) et de la contestation d'aides d'État (art. 88.2 CE). 
cipe d'interdiction des discriminations ${ }^{1}$. De même, en affirmant l'effet direct du droit communautaire, la Cour a permis aux particuliers de l'invoquer devant les juridictions nationales et de se prévaloir d'un grand nombre de droits individuels qui n'avaient pas été explicitement prévus par les traités. La Cour a aussi encouragé les juridictions nationales à contourner la Commission - qui détient le monopole de l'introduction des recours en manquement - en utilisant les questions préjudicielles pour mettre en évidence les cas d'incompatibilités des droits nationaux avec le droit communautaire. La jurisprudence de la Cour et du Tribunal a ainsi contribué à affirmer les droits des citoyens à un triple titre : la protection des droits fondamentaux, le droit à l'information et le principe de subsidiarité. Les juges ont favorisé l'accès des particuliers à une protection juridique communautaire, mais celle-ci est pour l'essentiel décentralisée dans les tribunaux nationaux et ne peut être considérée comme un instrument de contrôle des décisions et politiques de l'Union par les particuliers.

\section{L'ACCÈS DES CITOYENS AU PRÉTOIRE DE LA COUR : UNE APPROCHE PRATIQUE}

\section{LES INÉGALITÉS D' ACCÈS DES PARTICULIERS AU SYSTĖME JUDICIAIRE EUROPÉEN}

L'étude des particuliers auteurs de recours directs révèle de prime abord de forts contrastes quant à leur nationalité. Si l'on rapporte ce facteur à la population des États concernés, les plaignants belges, grecs et néerlandais se révèlent beaucoup plus nombreux que ceux d'Espagne, du Portugal, du Royaume-Uni et d'Italie. L'objet des recours et les traditions judiciaires nationales sont des éléments explicatifs pertinents, mais l'identité des plaignants a également son importance ; dans le nord de l'Europe ce sont surtout de grandes entreprises qui ont les moyens d'agir pour leur propre compte, tandis que dans le sud il s'agit davantage d'individus. En Grèce, en Italie et en Espagne on constate ainsi une nette disjonction entre le nombre d'affaires introduites et le nombre de plaignants impliqués, en raison de la tendance des petits agriculteurs et pêcheurs à introduire des recours collectifs.

Pour schématiser, on peut distinguer quatre types d'acteurs, dont les recours forment quatre catégories relativement homogènes. Un premier type de recours résulte de l'action collective d'un grand nombre d'acteurs qui partagent un intérêt commun ; c'est le cas, par exemple, des agriculteurs ou des pêcheurs des pays méditerranéens. Les recours qui visent de manière répétée un dossier unique sur une longue période forment une seconde catégorie ; ils émanent le plus souvent d'entreprises d'un même secteur qui cherchent à obtenir des différents États-membres qu'ils mettent leur législation en conformité avec le droit communautaire tel que l'interprète la Cour. Une troisième catégorie est constituée de recours introduits de manière réitérée par un plaignant qui tente de faire plier une institution européenne ou poursuit une stratégie de long terme, dont l'action judiciaire n'est qu'un élément. La dernière catégorie est celle des recours ponctuels et individuels, qui sont essentiellement introduits par des agriculteurs, des personnes physiques et des entreprises faisant appel de sanctions. La procédure de renvoi préjudiciel fait, elle aussi, l'objet d'utilisations très variables selon les États-membres, même si le système des renvois d'office limite les contrastes. En réduisant les données fournies par la Cour à la population de chaque État, on constate

1. Arrêt de la Cour, Van Gend en Loos, aff. 26/62, 1963, Rec. p. 1. 
que les citoyens luxembourgeois introduisent, en moyenne, vingt fois plus de recours préjudiciels que leurs voisins français.

De multiples facteurs expliquent ces contrastes nationaux. S'agissant des recours directs, on rappellera qu'ils constituent un moyen de dernière extrémité, dont les plaignants ne peuvent user que lorsque toutes les voies de recours nationales, y compris le renvoi préjudiciel, sont épuisées. Le besoin d'introduire un tel recours varie donc selon les caractéristiques de chaque système judiciaire national. Pour les recours préjudiciels, les asymétries nationales s'expliquent en grande partie par la nature des juridictions saisies des affaires (les cours constitutionnelles ne renvoyant pas, à l'exception de la Cour d'arbitrage belge), par leur attitude à l'égard du droit communautaire et par la qualité de la protection assurée par les voies de recours nationales. Plus largement, les traditions judiciaires et politiques des États-membres conditionnent étroitement la perception que les particuliers ont de l'intérêt d'une démarche auprès de la Cour de justice. Si dans certains États - comme en Allemagne - il existe une forte tradition d'utilisation des voies de recours judiciaires pour contester l'action des pouvoirs publics et une grande confiance dans la protection juridictionnelle des droits, dans d'autres - comme en France - le lobbying et les interventions auprès des responsables politiques seront préférés à l'action en justice. S'agissant des entreprises, Christopher Harding fait remarquer que le comportement judiciaire des dirigeants dépend de leur profil et que celui-ci varie beaucoup d'un État à l'autre '. Surtout, étant donné que la jurisprudence de la Cour sur l'accès des particuliers à son prétoire varie fortement selon les politiques communautaires, et que celles-ci ne concernent pas l'Union de manière homogène, des contrastes géographiques apparaissent nécessairement ${ }^{2}$. Enfin, le degré d'information des plaignants, de leurs avocats et des juges à l'égard des possibilités de recours est déterminant. On en veut pour preuve l'utilisation massive que les particuliers luxembourgeois et belges font des recours préjudiciels; la présence des institutions de l'Union à Bruxelles et Luxembourg les sensibilise assurément aux opportunités qu'offre l'intégration européenne et facilite leurs démarches. Le fait que le droit communautaire ait été enseigné dès les années 1950 dans les facultés de droit de ces pays n'y est pas étranger non plus.

Au-delà de ces variations nationales, il existe de forts contrastes dans l'accès des particuliers à la Cour de justice en fonction de leurs moyens et de leur connaissance du système judiciaire. Ces contrastes invitent à prêter attention aux visées stratégiques des plaignants, et à souligner les rapports étroits qui existent entre leurs motivations et la portée des recours. Les particuliers, en s'engageant dans des procédures judiciaires à l'échelle européenne, ne se contentent pas toujours de faire valoir leurs droits : ils peuvent également chercher à influer sur une politique communautaire ou à obtenir la modification de règles nationales. L'hermétisme de certaines dispositions du droit communautaire, originaire ou dérivé, ainsi que la jurisprudence parfois audacieuse de la Cour, encouragent de telles stratégies. Les recours peuvent aussi être mobilisés pour réclamer des dommages et intérêts substantiels à un État qui ne se serait pas conformé au droit

1. C. Harding, «Who Goes to Court in Europe ?..., art. cité, p. 106.

2. Les statistiques qui figurent dans le rapport annuel de la Cour au sujet de l'objet des recours directs des particuliers attestent du caractère très « sectorisé » de la jurisprudence. En 1999, les recours ont porté principalement sur les objets suivants (dans l'ordre décroissant) : rapprochement des législations, liberté d'établissement et services, agriculture, concurrence, environnement, fiscalité, dispositions sociales, statut des fonctionnaires et liberté de circulation des marchandises. CJCE, « Rapport annuel 1999 », Luxembourg, Office des publications officielles des Communautés européennes, p. 226. 


\section{La Cour de justice européenne}

communautaire '. Il arrive enfin que les recours directs des particuliers ne soient qu'un prétexte à de grandes batailles juridiques, qui impliquent directement ou indirectement - à travers la soumission d' « observations » à la Cour - des États-membres ou des institutions de l'Union ${ }^{2}$. C'est notamment le cas lorsque les juges sont appelés à se prononcer sur la compatibilité du droit national avec le droit communautaire.

Le recours préjudiciel fait, lui aussi, l'objet d'utilisations stratégiques. Lorsque les règles communautaires semblent plus favorables que les règles nationales, il est possible d'introduire un recours devant une juridiction nationale et de demander qu'elle forme un renvoi préjudiciel. Même si cette démarche ne suscite pas une intervention directe de la Cour de justice dans l'affaire, elle permet aux plaignants d'espérer que d'éventuels manquements au droit communautaire seront dénoncés ${ }^{3}$. Il importe donc d'envisager les interventions des particuliers devant la Cour, non seulement comme un moyen pour eux de défendre leurs droits, mais aussi d'exercer un contrôle plus large sur les activités des institutions, voire de participer à la gouvernance de l'Union.

\section{UNE TYPOLOGIE DES AUTEURS DE RECOURS DIRECTS}

L'état de la jurisprudence de la Cour et du 'Tribunal ainsi que la dimension stratégique des recours directs et préjudiciels expliquent que les prétoires communautaires sont occupés par des catégories d'acteurs très spécifiques. Cette situation apparaît clairement si l'on se penche sur l'identité des auteurs de recours en annulation. On peut, ici encore, proposer une typologie à trois entrées.

Un premier type de plaignants rassemble les groupes d'intérêts les plus puissants et les grandes entreprises qui ont les moyens nécessaires au suivi continu de la production normative de l'Union et des États-membres, et peuvent introduire des recours, quels qu'en soient la complexité, le coût, la durée et même le résultat. Les procédures sont en effet particulièrement lentes et lourdes : fin mars 2000 , la durée moyenne d'un recours direct devant le Tribunal était de dix-neuf mois ${ }^{4}$. Les divergences d'appréciation qui existent entre le Tribunal et la Cour encouragent par ailleurs, de plus en plus, les plaignants à contester les arrêts et ordonnances rendus en première instance : fin décembre 1999, $40 \%$ des recours directs faisaient 1'objet d'un pourvoi ${ }^{5}$, dont la durée moyenne était de vingt-deux mois. Les recours directs devant le Tribunal sont en outre particulièrement hasardeux ; en 1999, sur 227 arrêts et ordonnances (non comprises les affaires jointes), le Tribunal n'a déclaré les recours fondés ou partiellement fondés que dans vingt-trois cas. Les renvois préjudiciels sont eux aussi assortis de délais importants : en 2000 , leur durée moyenne était de près de vingt-quatre mois - alors qu'ils ne représentent qu'une des phases de processus judiciaires plus complexes. Les

1. C. Barnard, «A European Litigation Strategy: The Case of the Equal Opportunities », dans J. Shaw, G. Morre (eds), Legal Dynamics of European Union, Oxford, Clarendon Press, 1995 , p. 253-272. 149.

2. R. Dehousse, «L'Europe par le droit », Critique internationale, 2, hiver 1999, p. 133-

3. R. Dehousse, «L'Europe par le droit : plaidoyer pour une approche contextuelle», Politique européenne, 1, avril 2000, p. 63-71.

4. Greffe de la Cour de justice, «Statistiques judiciaires : état au 31 mars 2000 », Luxembourg, 4 avril 2000.

5. Seuls $31 \%$ des décisions attaquables du Tribunal faisaient l'objet de pourvois fin décembre 1998 et $25 \%$ un an plus tôt. En 1999, sur cinquante-sept recours, la Cour a annulé huit arrêts ou ordonnances du Tribunal. 


\section{Olivier Costa}

délais de jugement et les faibles chances de succès expliquent que les grands lobbies et entreprises représentent une forte proportion des plaignants.

Le coût des procédures est lui aussi dissuasif. Le recours à un avocat spécialisé est indispensable, et revient à environ 2500 euros pour une affaire simple. Dès qu'il est question de contester une règle nouvelle ou de soulever un motif d'annulation jamais examiné par le Tribunal, il faut faire appel à des experts. Les procédures consistent en effet, pour l'essentiel, en des échanges répétés de mémoires très techniques. Pour les affaires de concurrence, ces documents comptent souvent plus de cent pages et se multiplient : requête d'origine, mémoire de défense, réplique du plaignant, interventions éventuelles d'autres acteurs concernés, répliques à ces interventions, mémoires relatifs à des incidents de procédure... À son terme, une affaire complexe peut occasionner plus de 150000 euros de frais d'avocats. Un plaignant débouté peut aussi être condamné aux dépens qui, pour une affaire importante, se montent aisément à 100000 euros. Les juges prennent toutefois en compte la situation financière du particulier, sa bonne foi et le degré de responsabilité de l'institution mise en cause, sauf lorsqu'il s'agit de recours manifestement dilatoires, aucunement fondés en droit, à l'égard desquels les juges sont désormais très fermes.

Si les délais, coûts et risques d'un recours direct sont de nature à dissuader des individus ou des petites entreprises, il en va tout autrement pour les grands groupes et les lobbies les plus puissants. Leur capacité à affronter ces contraintes ne l'explique qu'en partie ; il faut aussi constater que leurs critères de réussite d'une action en justice incorporent d'autres enjeux qu'une décision favorable '. Ces acteurs peuvent essayer de provoquer un débat public pour soutenir d'autres démarches entreprises afin d'obtenir l'inflexion d'une politique ou la modification d'une norme, ou encore chercher à susciter une jurisprudence abondante pour faire évoluer l'interprétation de règles communautaires.

Une seconde catégorie de plaignants est formée des acteurs issus de catégories professionnelles bien déterminées. Ils ne jouissent pas nécessairement de ressources financières et juridiques importantes, mais sont mobilisés par des précédents qui ont été favorables à des particuliers dont la situation était proche de la leur, et sont de ce fait bien informés des possibilités ouvertes par un type précis de recours. L'examen de la jurisprudence du Tribunal montre que, tous les ans, des acteurs appartenant à un ou plusieurs groupes économiques ou sociaux restreints introduisent massivement des recours sur la base d'un précédent jurisprudentiel ou d'une situation exceptionnelle au regard du droit communautaire. Ces dernières années, ce fut successivement le cas des viticulteurs des pays du Sud, des petits garagistes, des producteurs de lait, des agents en douanes et des gérants de stations-service néerlandais ${ }^{2}$. Même si ces plaignants disposent de ressources financières réduites et ne sont pas familiers du microcosme communautaire, ils connaissent les possibilités offertes par un type précis de recours. Les syndicats et les associations professionnelles jouent souvent un rôle clé dans la constitution de dossiers «standardisés», qui représentent l'essentiel des recours introduits par ces acteurs. Ceux-ci, à défaut de moyens importants, acquièrent l'expertise collective nécessaire à l'investissement d'une « brèche » ouverte par une décision du Tribunal ou de la Cour.

1. M. Galanter, «Why the "Haves" Come out Ahead : Speculations on the Limits of Legal Change », Law and Society, 1974, p. 95-160.

2. Les producteurs de lait, qui ont massivement introduit, depuis le début des années 1990, des recours contre des décisions de la Commission relatives aux quotas, étaient encore à l'origine de 190 affaires en 1998, et de 88 en 1999. 
Les individus constituent une troisième catégorie de plaignants. Compte tenu des contraintes de délais et de coût qui s'imposent à eux comme aux autres, ils sont peu nombreux ${ }^{1}$. Si l'on exclut le cas des fonctionnaires européens, le nombre de recours formés par des individus est de l'ordre de quelques dizaines par an, à comparer aux 8396 requêtes dont la Cour européenne des droits de l'homme a été saisie en 1999. Les juges européens font valoir que les décisions des institutions européennes concernent rarement des individus en particulier, qui ont donc peu de motifs d'introduire des recours. Il faut toutefois noter que, dans les rares secteurs où les juges ont assoupli les conditions de recevabilité des recours des particuliers (tel que l'accès aux documents des institutions communautaires), ceux-ci tirent largement profit de l'opportunité. On soulignera également le peu d'empressement que les juges mettent à accueillir les recours des individus dans les quelques domaines où un contentieux se développe autour de règles communautaires qui leur sont directement destinées. C'est notamment le cas, depuis 1998, des règles relatives à la libre circulation des personnes, qui ont amené la Cour à évoquer le principe de citoyenneté de l'Union ${ }^{2}$. Pour l'heure, les juges se sont montrés particulièrement prudents et n'ont rien fait pour inciter les citoyens à agir auprès d'eux.

Au constat de la faiblesse numérique des recours introduits par des individus, i1 faut ajouter celui d'un certain élitisme, les plaignants appartenant pour la plupart à une minorité de citoyens parfaitement informés des voies de recours et évoluant dans le microcosme communautaire. La Cour s'est doté d'un système d'assistance judiciaire complet $^{3}$ et le plaignant indigent peut échapper aux dépens même s'il est débouté, pour peu qu'il fasse preuve de bonne foi. Ces facilités sont toutefois loin de compenser les faiblesses de l'information des particuliers et des avocats non spécialistes de droit européen sur les possibilités de recours et les conditions de leur introduction. Le contentieux qui se développe en matière d'accès aux documents du Conseil et de la Commission atteste de cette situation. Les recours formés par des individus émanent principalement de journalistes ${ }^{4}$ et de responsables politiques. Le nombre de députés européens qui introduisent des recours ou sollicitent la formation d'un renvoi préjudiciel est d'ailleurs frappant ${ }^{5}$.

1. Le Tribunal et la Cour ne distinguant pas les personnes physiques des personnes morales dans leurs bases de données, il est délicat de rassembler des chiffres à cet égard. La rédaction des arrêts et ordonnances permet toutefois de distinguer ces deux catégories de plaignants par l'emploi des formules «établi à... » pour les personnes morales et « demeurant à... » pour les personnes physiques.

2. Cf. notamment : arrêt de la Cour, Martinez Sala, C-85/96, 12 mai 1998, Rec. p. I-2691 ; arrêt de la Cour, Wijsenbeek, C-378/97, 21 septembre 1999, Rec. p. I-6207.

3. L'article 76 du règlement de procédure de la Cour prévoit l'assistance judiciaire gratuite, pour laquelle il existe un budget limité. Chaque partie peut, à tout moment, demander à en bénéficier si elle se trouve « dans l'impossibilité de faire face en totalité ou en partie aux frais de l'instance ». Si la demande précède le recours, la partie doit en exposer l'objet pour permettre à la Cour d'examiner le bien-fondé de l'action. Pour présenter la demande, la partie n'est pas obligée de se faire représenter par un avocat. L'octroi de l'assistance judiciaire gratuite n'exempte pas d'une éventuelle condamnation aux dépens; la Cour peut en outre récupérer les sommes versées.

4. Cf., par exemple, l'arrêt du Tribunal, Svenska Journalistförbundet v. Conseil, T-174/95, 17 juin 1998, Rec. p. II-2289.

5. L'arrêt Wijsenbeek précité résulte d'un renvoi préjudiciel, dans le cadre d'une procédure pénale engagée contre un député européen qui, de retour de session à Strasbourg, avait refusé de présenter son passeport et d'établir sa nationalité néerlandaise lors de son entrée aux Pays-Bas. Cf. également l'arrêt du Tribunal Hautala v. Conseil, T-14/98, 19 juillet 1999, Rec. p. II-2489. 
L'assiduité - relative - des acteurs rompus aux subtilités du droit communautaire auprès de la Cour n'est pas seulement le produit de leur expertise, mais aussi des motifs particuliers qui les animent. De même que les grandes entreprises peuvent déployer des stratégies qui font peu de cas de la durée et du coût des procédures, voire de leur issue, les individus présents devant la Cour ont souvent des motivations autres que l'obtention de l'annulation d'un acte. L'introduction d'un recours peut constituer un geste politique. Le député européen français Georges Berthu, membre du groupe eurosceptique «Europe des Nations », demanda ainsi l'annulation du traité de Maastricht au motif qu'il attentait, selon lui, à la souveraineté de la République française ${ }^{1}$. De la même manière, il tenta de s'opposer en 1997 à l'introduction de l'euro ². Dans les deux cas, il ne se faisait certainement pas d'illusions quant aux résultats juridiques de son action. La jurisprudence de la Cour relative à l'accès aux documents et à la libre circulation montre clairement que les individus impliqués cherchent avant tout à contester le principe de certaines règles ou à dénoncer leur mauvaise application. Plus largement, leurs interventions sont souvent sous-tendues par une contestation du mode de fonctionnement du système institutionnel de l'Union.

Pour être exhaustif, précisons que les voies de recours sont également empruntées par deux autres types d'acteurs. Les premiers peuvent être rangés dans la catégorie des « procéduriers »; leurs recours, qui portent généralement sur des questions extérieures aux compétences de la Cour et de l'Union, sont souvent étudiés de manière expéditive, les juges européens refusant de s'ériger en juges de paix ou d'épuiser les ressources de l'institution dans l'étude de recours sans fondement. Certains plaignants peuvent, en second lieu, être assimilés à des «prête-noms » qui agissent pour le compte d'organisations professionnelles, d'associations de consommateurs ou de lobbies ; ceux-ci cherchent à multiplier les recours pour façonner la jurisprudence, mais ne peuvent le faire eux-mêmes en raison de l'attitude restrictive de la Cour sur ce point ${ }^{3}$.

\section{LES PERSPECTIVES DE L'INTÉGRATION EUROPÉENNE ET L'ACCÈS DES CITOYENS AU JUGE COMMUNAUTAIRE}

La possibilité pour les particuliers, et notamment pour les individus, d'agir devant la Cour pour défendre leurs droits ou peser sur le fonctionnement du système politique de l'Union est au cœur d'une double contradiction. La première oppose le discours que tiennent les juges de Luxembourg sur l'intervention des citoyens auprès d'eux à leur jurisprudence et à leurs préoccupations quant à l'avenir du système judiciaire européen. La seconde oppose cette jurisprudence et ces préoccupations aux intentions et ambitions des représentants des États-membres quant à l'accès des citoyens au droit communautaire.

1. Ordonnance du Tribunal, Berthu v. Conseil, T-207/97, 12 mars 1998, Rec. p. II-0509.

2. Ordonnance du Tribunal, Berthu v. Commission, T-175/96, 15 mai 1997, Rec. p. II-811.

3. Les auteurs évoquent souvent le cas de l'arrêt Defrenne (aff. 80/70 et 43/75), pour lequel une plaignante fut choisie par des groupes féministes afin d'introduire un recours, sans qu'elle soit jamais réellement impliquée dans l'affaire. Cf. C. Harlow, R. Rawlings, Pressure through Law, Londres, Routledge, 1992. 
Il existe un fort hiatus entre le discours que tiennent les juges et les fonctionnaires de la Cour au sujet de la contribution de leur institution à la défense des intérêts des citoyens et, partant, à l'affirmation de l'Union comme un système respectueux des principes démocratiques, et les préoccupations qu'ils ont pu exprimer ces dernières années quant à la réforme des compétences et du mode de fonctionnement de leur institution. Malgré les exigences que la Cour formule à l'endroit des systèmes judiciaires nationaux en matière d'accès à la justice, et malgré son discours sur sa participation à la défense des droits des individus, la question des relations entre le système politique et juridique de l'Union et les particuliers, de même que celle des garanties juridictionnelles qui leur sont offertes à l'échelle supranationale, sont absentes des documents de réflexion que la Cour a produits ces dernières années au sujet de son avenir ' ${ }^{\text {. Leur lec- }}$ ture montre que les juges et les experts de la Cour se préoccupent avant tout de préserver et d'améliorer la capacité de fonctionnement de l'institution et, implicitement, d'affirmer son rang d'instance suprême du système juridictionnel européen, dans un rapport clairement hiérarchique vis-à-vis des cours nationales ${ }^{2}$. Ils mettent ainsi l'accent sur les enjeux fonctionnels d'une réforme de l'organisation de la Cour. Selon eux, elle doit répondre prioritairement au déséquilibre structurel qui existe entre le volume d'affaires entrantes et les capacités de l'institution. La charge de travail de la Cour ira en effet croissant en vertu de l'extension de ses compétences juridictionnelles par les traités d'Amsterdam et de Nice, des conventions adoptées dans le cadre de la coopération policière et judiciaire en matière pénale, de la place grandissante que le droit communautaire occupe dans la vie des citoyens et des entreprises communautaires, et des perspectives d'élargissement de l'Union. Les négociateurs du traité de Nice ont partiellement répondu à ces préoccupations en adoptant une profonde réforme de l'organisation de la juridiction communautaire, qui vise à simplifier son mode de fonctionnement et à réduire ses délais de jugement ${ }^{3}$.

Les juges communautaires donnent également la priorité au pragmatisme et à l'efficacité fonctionnelle lorsqu'ils se penchent sur les détails de l'organisation interne de la Cour et du Tribunal. Ils ont obtenu du Conseil qu'il prenne - sans attendre la réforme des traités - plusieurs mesures afin d'introduire le principe du juge unique au Tribunal, de transférer des compétences de la Cour vers celui-ci, et de faciliter la hié-

1. Entre autres : CJCE, « Document de réflexion de la Cour de justice et du Tribunal sur l'avenir du système juridictionnel de l'Union », présenté au Conseil des ministres de la Justice les 27 et 28 mai 1999 ; "Propositions présentées par la Cour et le Tribunal pour le nouveau contentieux de la propriété intellectuelle $», 2000$. Dans le même sens, cf. les réponses du membre français de la Cour de justice Jean-Pierre Puissochet : «Entretien croisé des juges français », Pouvoirs, 96, janvier 2001, p. 174-175.

2. Sur ce point, cf. T. Ronse, D. Waelbroeck, « La Cour de justice, juridiction suprême », dans P. Magnette, E. Remacle, Le nouveau modelle européen. Vol. 1. Institutions et gouvernance, op. cit., p. 89-103.

3. Selon le traité de Nice, la Cour de justice restera formée d'un juge par État-membre, mais elle comprendra désormais plusieurs formations. Elle pourra siéger en chambres (composées de trois ou cinq juges), en grande chambre (composée de onze juges) ou en assemblée plénière. Le Conseil, statuant à l'unanimité, pourra augmenter le nombre des avocats généraux, qui reste fixé à huit. Les compétences du Tribunal seront élargies, notamment à certains recours préjudiciels. En contrepartie, des chambres juridictionnelles pourront lui être adjointes pour connaître en première instance de certaines catégories de recours. Une déclaration prévoit la création rapide d'une chambre juridictionnelle compétente pour statuer en première instance sur les litiges entre la Communauté et ses agents. 


\section{Olivier Costa}

rarchisation des affaires en fonction de leur complexité et de leur degré d'urgence. Le Tribunal a également bénéficié du droit de réviser lui-même son règlement, et s'est saisi de cette possibilité pour accélérer les procédures par un ensemble de modifications ${ }^{1}$. Ces réformes n'ont pas été sans conséquences pour les particuliers, puisqu'elles font toutes primer l'efficacité et la rapidité de la décision sur les droits des parties à défendre leurs positions respectives. La Cour et le Tribunal sont animés par un souci similaire lorsqu'ils s'adressent aux justiciables, qui sont priés de contribuer au déroulement « rapide et efficace » des procédures, et invités à limiter la longueur et la complexité des mémoires et des plaidoiries ?

On constate plus largement que l'ensemble des textes, projets et rapports de la Cour ont pour point commun de ne jamais faire référence aux «citoyens » ou aux « individus » ${ }^{3}$. Ce manque d'intérêt des juges pour ces acteurs éclaire d'une lumière particulière le décalage qui existe entre les possibilités concrètes d'accès des citoyens à la Cour de justice, et les exigences que celle-ci formule quant à leur accès au juge national. Il est tentant de considérer que, si les juges de Luxembourg ont reconnu aux particuliers un droit à la protection juridictionnelle, c'est avant tout pour assurer la mise en œuvre effective et uniforme du droit communautaire dans les États-membres et pour garantir sa primauté.

\section{LA RESTRICTION DE L'ACCÈS DES PARTICULIERS AU JUGE COMMUNAUTAIRE : UN ANACHRONISME?}

Face aux perspectives d'engorgement des prétoires communautaires, il semble que les préoccupations fonctionnelles d'efficacité et de rapidité de la décision aient pris le pas sur la volonté de la Cour de s'affirmer comme la garante des droits fondamentaux des citoyens européens. Les commentateurs avaient pourtant été nombreux à interpréter en ce sens l'avis 2/94 de la Cour, qui excluait que l'Union puisse adhérer, sans révision préalable de ses traités, à la Convention européenne des droits de l'homme ${ }^{4}$. Les juristes les plus avertis avaient estimé qu'il s'agissait d'une tentative des juges communautaires de sortir de leur rôle exclusivement économique et d'investir de manière plus autonome le terrain des droits de l'homme. Ce constat $n$ 'a pas connu de traduction concrète dans la jurisprudence et le discours des juges, même si la perspective de l'avènement d'un « double standard » en matière de défense des droits de l'homme ${ }^{5}$ a été confirmée à deux titres.

1. JOCE L 322 du 19 décembre 2000.

2. Cf. sur le site Internet de la Cour la «note destinée à servir de guide aux conseils des parties lors de l'audience des plaidoiries» et la «note pour la rédaction des mémoires» (<www.curia.eu.int $>$ ).

3. Les termes « citoyen » et « individu» sont, d'ailleurs, absents des statuts de la Cour et de son règlement de procédure ; dans ce dernier, la mention « particulier » n'apparaît qu'à une reprise, en relation avec la possibilité de l'attribution d'une aide judiciaire par la Cour (article 104§6). Le terme consacré est « personne », le règlement n'introduisant la distinction entre personne «physique » et « morale » qu'en un seul endroit.

4. CJCE, avis 2/94 du 28 mars 1996, Rec., I, p. 1763. Cf. J.-F. Flauss, dans Les Petites Affiches, 91, 1997, p. 4 ; P. Wachsmann, dans Revue trimestrielle de droit européen, 1996, p. 467.

5. L'expression est de Denys Simon : «Des influences réciproques entre CJCE et CEDH. “je t'aime, moi non plus ?" », Pouvoirs, 96, janvier 2001, p. 31-50. Cf. aussi V. Constantinesco, «Le renforcement des droits fondamentaux dans le traité d'Amsterdam », Le traité d'Amsterdam, réalités et perspectives, Paris, Pedone, 1999, p. 33 et suiv. 
Elle l'a été une première fois par le traité d'Amsterdam, qui a introduit dans l'article 6 du traité sur 1'Union la formule suivante : "L'Union est fondée sur les principes de la liberté, de la démocratie, du respect et des droits de l'Homme et des libertés fondamentales, ainsi que de l'État de droit, principes qui sont communs aux États-membres ». Cette affirmation, loin d'être purement symbolique, marque une rupture avec la situation préalable qui pouvait laisser penser que l'Union abandonnait la garantie des droits fondamentaux aux États-membres et au Conseil de l'Europe. Les standards de protection offerts par le Conseil de l'Europe ont toutefois souffert de son élargissement rapide vers des pays où la situation des droits de l'homme demeure problématique (Croatie, Russie, Ukraine...). Afin de se prémunir contre toute dérive, le traité d'Amsterdam a donc énoncé les valeurs de l'Union et introduit à l'article 7 du traité sur l'Union un mécanisme permettant de sanctionner la « violation grave et persistante par un État-membre de principes énoncés à l'article $6 »$.

La formalisation des principes fondateurs de l'Union a également été guidée par l'évolution des rapports entre les ordres juridiques nationaux et communautaire. Compte tenu de l'élévation globale du degré d'exigence dans la protection des droits fondamentaux en Europe occidentale, il n'était pas admissible que le droit communautaire échappe à un tel contrôle. L'arrêt de la Cour constitutionnelle allemande au sujet du traité de Maastricht avait clairement stigmatisé les limites d'un système politique qui ne dispose pas d'un législateur pleinement représentatif et qui restreint fortement I'accès des particuliers au juge '. Cette influence des juges nationaux n'est d'ailleurs pas nouvelle : l'engagement de la Cour de justice dans la protection des droits fondamentaux résulte en partie de ce que les juridictions nationales (notamment allemandes et italiennes) n'ont accepté de reconnaître la primauté du droit communautaire qu'à la condition que celui-ci respecte les droits fondamentaux. Le traité d'Amsterdam ne se contente donc pas, comme l'avait fait le traité de Maastricht, d'un aggiornamento destiné à intégrer la jurisprudence de la Cour, mais offre des possibilités d'évolution considérables ${ }^{2}$. L'assimilation de l'Union à un « espace de liberté, de sécurité et de justice » est en particulier une invitation pour la Cour à prendre pleinement en charge la protection des droits fondamentaux, dans le cadre d'un espace judiciaire unique.

L'hypothèse de l'introduction d'un « double standard » en matière de défense des droits de l'homme a été confirmée en juin 1999 par la décision du Conseil européen de Cologne d'établir un «catalogue» des droits fondamentaux dans 1'Union. La Charte des droits fondamentaux, qui est la première traduction concrète des réformes introduites par le traité d'Amsterdam, vise à éviter toute dérive élitaire du système de protection des droits, et à faire connaître aux citoyens les droits qu'ils pourront invoquer dans la perspective d'une «autonomisation» de la protection des droits de l'homme par l'Union. Les négociateurs de la Charte étaient nombreux à espérer que, grâce à l'inscription de ce texte dans les traités, la Cour accèderait au statut d'instance de protection des droits individuels dans l'Union. Toutefois, les représentants des États-membres réunis à Nice en décembre 2000 ont renoncé à donner à ce texte un caractère contraignant. Cette situation n'interdit pas aux juges communautaires de

1. Cf. J. Gerkrath, «La critique de la légitimité démocratique de l'Union européenne selon la Cour constitutionnelle fédérale de Karlsruhe », dans G. Duprat (dir.), L'Union européenne, droit, politique, démocratie, op. cit., p. 209-241.

2. Sur ce point, cf. les analyses proposées par le rapport Quermonne (« Le bouleversement des perspectives juridiques », p. 96 et suiv.), dans L'Union européenne en quête d'institutions légitimes et efficaces. Rapport du groupe de réflexion sur la réforme des institutions européennes, Paris, La Documentation française, 1999. 
faire référence à certaines dispositions de la Charte, mais, tant que celle-ci n'aura pas force de loi, elle ne sera probablement invoquée que dans le respect du droit communautaire originaire et dérivé existant et, plus largement, dans le respect de ce que les juges pensent être la nature fondamentale du droit communautaire. Or, comme on l'a indiqué, ils semblent pour l'heure davantage préoccupés par la préservation de leur capacité à exercer leurs compétences que par l'extension de celles-ci. L'amélioration des relations entre la Cour de justice des Communautés et la Cour européenne des droits de l'homme est le signe le plus probant de la priorité que les juges de Luxembourg accordent à la consolidation de l'efficacité fonctionnelle de leur institution '.

Les évolutions récentes des traités et, plus largement, les ambitions que manifestent les représentants des États-membres à l'égard de l'espace judiciaire européen, s'accordent mal avec les restrictions persistantes que les juges communautaires apportent à l'accès des individus à leurs prétoires. L'argumentaire de la Cour est pourtant d'une fragilité croissante : seuls quelques juges persistent d'ailleurs à le défendre ouvertement $^{2}$. Il ressort de l'étude de la jurisprudence de la Cour, de la lecture des écrits des juges et de la conduite d'entretiens avec divers acteurs de l'institution, que ce discours justificatif s'articule autour de cinq arguments principaux. Le premier est celui de la spécificité de l'ordre juridique communautaire : compte tenu de l'absence de distinction entre loi et règlement, l'attribution d'un droit de contrôle généralisé aux particuliers sur les règlements communautaires (au sens où ils sont définis par le traité $\mathrm{CE}$ ) équivaudrait à leur donner la possibilité de déclencher un contrôle de constitutionnalité ${ }^{3}$. Or rares sont les États-membres qui permettent aux particuliers de contester des actes législatifs autrement que par la voie de l'exception d'illégalité. Les juges avancent en second lieu que la Communauté met rarement sa législation en œuvre elle-même et que les intérêts des particuliers ne sont, dans la grande majorité des cas, affectés que par les mesures d'exécution des États-membres, susceptibles de recours au niveau national. Ils soulignent aussi que la procédure de renvoi préjudiciel permet au juge national de s'assurer de la légalité d'une mesure communautaire. Les juges invoquent en quatrième lieu la lettre des traités : ils estiment qu'il ne leur revient pas de décider unilatéralement d'ouvrir plus largement leurs prétoires aux individus. Enfin, ils avancent que les citoyens ont, pour l'heure, peu de raisons d'agir devant la Cour, le droit communautaire concernant avant tout les opérateurs économiques. Leur participation au contentieux communautaire est donc certes embryonnaire, mais elle est appelée à se développer au rythme du renforcement de la citoyenneté européenne et du développement de nouvelles politiques tournées vers les individus.

Les arguments de la Cour et du Tribunal sont d'une fragilité croissante. L'invocation de la compétence de droit commun des cours nationales n'est pas très satisfaisante, compte tenu de la multiplication des situations dans lesquelles le droit communautaire

1. Cf. le numéro de la revue Pouvoirs consacré aux Cours européennes de Luxembourg et Strasbourg, 96, janvier 2001, et en particulier l'article précité de Denys Simon.

2. Sur ce point, cf. A. Dashwood (ed.), Reviewing Maastricht: Issues for the 1996 IGC, Londres, Sweet \& Maxwell, 1996, p. 308-311.

3. La protection des droits des individus est un des éléments qui ont motivé les réflexions relatives à la création d'une nouvelle catégorie d'actes, la « loi communautaire ». Ce processus a été entamé au sein du Parlement européen, à l'occasion de la rédaction du projet de traité d'Union adopté le 14 février 1984 (projet Spinelli). La question a ressurgi lors des négociations sur 1'Union; faute de consensus, les rédacteurs du traité de Maastricht se sont contentés d'inviter la prochaine conférence intergouvernementale à envisager l'introduction d'une hiérarchie des normes communautaires (déclaration $n^{\circ} 16$ ). Comme on le sait, cette invitation est restée sans suite. 
produit directement ses effets pour des particuliers. On constate en outre une inflation des cas où les cours nationales et européenne se déclarent également incompétentes, les premières renvoyant au texte communautaire et la seconde aux mesures nationales prises en application de celui-ci. S'agissant de la possibilité pour les citoyens de demander un renvoi préjudiciel, on notera que, sauf en dernière instance, la décision de le former appartient au juge national saisi, ce qui est la source de fortes distorsions. En ce qui concerne la lettre des traités, rappelons que les juges communautaires ont parfois pris certaines libertés à son égard, notamment lorsqu'ils ont attribué au Parlement européen la capacité d'introduire des recours '. Quant à l'absence d'intérêt à agir des particuliers, on rappellera que les arrêts les plus récents de la Cour et du Tribunal relatifs à la citoyenneté européenne montrent que les juges sont peu pressés d'encourager les individus à réclamer auprès d'eux l'application du droit communautaire.

Selon la grande majorité des auteurs ${ }^{2}$, la limitation de l'accès des particuliers au prétoire communautaire pose donc le problème d'un système politique qui, bien que fondé sur la règle de droit, $n$ 'assure pas la soumission des activités de ses institutions législatives et exécutives à un contrôle juridictionnel ou, du moins, le fait dans des conditions excessivement restrictives. Ces auteurs soulignent que les institutions communautaires prennent un nombre croissant de mesures et de sanctions qui sont applicables sans intervention des autorités nationales. En pareil cas, en l'absence de possibilité de recours direct, le droit au juge n'est plus garanti. Deux solutions à cette situation sont fréquemment avancées : la première serait l'adhésion de la Communauté à la Convention européenne des droits de l'homme; la seconde consisterait à énoncer clairement les droits fondamentaux et à assouplir les conditions d'introduction d'un recours direct pour permettre aux citoyens de s'en prévaloir devant la Cour. D'aucuns appellent ainsi à une ouverture des possibilités de recours direct, voire à une association plus étroite des citoyens à la prise de décision dans l'Union par ce biais, et considèrent la possibilité de former des recours directs devant la Cour de justice comme un droit fondamental et un élément essentiel de la citoyenneté européenne ${ }^{3}$.

1. La Cour a progressivement reconnu au Parlement européen le droit de former divers types de recours, sans que cela soit prévu par les traités, et obtenu des États-membres qu'ils suivent son raisonnement et modifient l'article 173 CEE à l'occasion de la rédaction du traité de Maastricht. Ce processus, dit de « légitimation active » du Parlement européen, a fait l'objet d'une abondante littérature juridique. Cf., entre autres, J.-F. Chambault, «L'ouverture du recours en annulation au Parlement européen : aboutissement et cohérence d'une décennie de jurisprudence », Revue $d u$ marché commun et de l'Union européenne, 343, janvier 1991, p. 40-48.

2. C'est le cas de la plupart des manuels de droit communautaire et des monographies consacrées à la Cour de justice. Cf. aussi G. Vandersanden, «Pour un élargissement du droit des particuliers d'agir en annulation contre des actes autres que les décisions qui leur sont adressées », Cahiers de droit européen, 1995, p. 535-560 ; J. Dutheil de la Rochère, «Droit au juge, accès à la justice européenne », op. cit. Sur ce point, le Rapport Quermonne est particulièrement explicite : «Le régime du recours en annulation contre les règlements souffre actuellement la critique dans la mesure où les particuliers ne sont recevables à intenter un tel recours que dans des conditions très restrictives puisqu'ils doivent faire état d'une situation qui les caractérise individuellement par rapport à ce règlement. En fait seuls les institutions et les États-membres peuvent attaquer les règlements. Cette situation qui réduit la protection juridique due aux particuliers n'est pas acceptable »: L'Union européenne en quête d'institutions légitimes et efficaces, op. cit., p. 116. Les auteurs soulignent.

3. G. Vandersanden, « Pour un élargissement du droit des particuliers d'agir en annulation contre des actes autres que les décisions qui leur sont adressées », art. cité, et $G$. Vandersanden (dir.), La réforme du système juridictionnel communautaire, Bruxelles, Éditions de l'Université de Bruxelles, 1994. 
Comment expliquer alors l'attitude persistante des juges de Luxembourg ? Certains d'entre eux reconnaissent que leur position n'est pas seulement motivée par un raisonnement juridique, mais aussi par les contraintes qui pèsent sur le fonctionnement de la Cour. On a indiqué précédemment à quel point ils étaient soucieux de préserver la capacité de leur institution à remplir ses missions efficacement. Il faut par ailleurs remarquer que les juges communautaires ne sont plus systématiquement de fervents europhiles, issus de cercles œuvrant pour l'intégration européenne ; il s'agit de plus en plus de magistrats de carrière qui s'en tiennent au droit et privilégient une approche «textualiste » des traités et du droit dérivé. Ceci est surtout vrai pour le Tribunal, et explique pour partie les divergences qui existent entre sa jurisprudence et celle de la Cour quant aux conditions d'ouverture des recours directs ${ }^{1}$.

Au-delà de ces considérations juridiques et fonctionnelles, on peut entrevoir des motifs politiques et stratégiques à l'attitude des juges. Leur jurisprudence restrictive doit être analysée comme un reflet de la conception qu'ils ont de leurs rapports avec les autres institutions et de la place de la Cour dans le système politique de l'Union. À cet égard, leur logique n'est pas nécessairement celle de « l'activisme judiciaire » ${ }^{2}$. II n'est plus possible, comme ce fut le cas dans les années 1960 et 1970, de considérer que la jurisprudence de la Cour est tout entière déterminée par le souci d'une intégration communautaire plus étroite et d'un renforcement de l'échelon européen de gouvernement. Face à un activisme bien réel, certains auteurs ont estimé que les «Pères fondateurs » avaient introduit dans le traité de Rome un « code génétique » propre à inciter la Cour à promouvoir, quelles que soient les circonstances, une lecture fédérale de l'intégration européenne $^{3}$. Sa jurisprudence à l'égard des particuliers montre toutefois que la Cour peut aussi souscrire à une logique de self restraint, et ce, pour diverses raisons. Le contexte politique a, en premier lieu, un impact certain sur les décisions des juges ; les réticences qui entourent la construction européenne dans les opinions publiques depuis le début des années 1990 sont, de toute évidence, peu propices aux audaces jurisprudentielles. Les contraintes institutionnelles sont un second facteur : les juges doivent notamment veiller à préserver le consensus interne à la Cour, puisqu'ils n'ont pas la possibilité d'exprimer des positions minoritaires comme c'est le cas à la Cour européenne des droits de 1'homme. En troisième lieu, les juges communautaiues manifestent une forte aspiration à l'《exclusivité »: malgré l'engorgement de leur institution et son absence de moyens déconcentrés dans les États-membres, il ressort clairement de leurs arrêts qu'ils tiennent à rester les seuls interlocuteurs des plaignants ou, du moins, à décider en dernier ressort. Les juges défendent, plus ou moins ouvertement, un schéma dans lequel le Tribunal serait une juridiction d'appel pour les cours nationales, ce qui implique la restriction du droit d'accès des citoyens à son prétoire. La Cour et le Tribunal s'abstiennent d'ailleurs de tout arrêt de principe en la matière et limitent l'effet rétroactif de certains arrêts d'annulation afin d'éviter une multiplication des actions. Plus encore, ils vont

1. En 1998, la Cour a, par exemple, annulé à quatre reprises des arrêts par lesquels le Tribunal avait déclaré irrecevables des recours en annulation formés par des sociétés contre des décisions de la Commission, au motif que les plaignants n'étaient pas directement concernés. Dreyfus/Commission, C-386/96 P, Rec. p. I-2309; Compagnie continentale (France)/Commission, C-391/96 P, Rec. p. I-2377 ; Glencore Grain/Commission, C-403/96 P, Rec. p. I-2405 et C-404/96 P, Rec. p. I-2435.

2. Pour une critique des usages abusifs de cette notion, cf. D. Keeling, « In Praise of Judicial Activism. But What Does it Mean? And Has the European Court of Justice Ever Practised it ? », dans Scritti in onore di Giuseppe Federico Mancini, Milan, A. Giuffrè, 1998, p. 505-536.

3. G. F. Mancini, D. T. Keeling, «Democracy and the European Court of Justice», Modern Law Review, 57, 1994, p. 186. 
jusqu'à revenir sur des jurisprudences antérieures, dont ils estiment qu'elles suscitent un nombre excessif de recours ${ }^{1}$.

La jurisprudence de la Cour de justice, contrairement à celle de nombre de cours nationales, ne s'expliquerait ainsi pas tant par la recherche du pouvoir en soi, que par le souci de garantir la cohérence du droit communautaire et, ce faisant, de servir les objectifs des traités. Le rôle clé que le droit communautaire a joué dans le processus d'intégration européenne incite les juges à donner la priorité à la préservation de sa force. À cet égard, la restriction du droit de recours des citoyens est particulièrement importante. Elle permet, d'une part, d'éviter l'affaiblissement du droit communautaire par la remise en cause d'un nombre croissant de décisions et, d'autre part, de limiter les interventions de la Cour, et donc de la faire échapper à la dénonciation récurrente du « gouvernement des juges ».

L'accès des personnes physiques au prétoire de la Cour de justice est particulièrement restreint. Il l'est tellement que Lionel Jospin, dans son discours du 28 mai 2001 sur «l'avenir de l'Europe », estimait que « la Cour européenne de justice devrait pouvoir être saisie, dans certaines conditions, directement par les citoyens », en laissant entendre que tel n'est pas le cas actuellement. II faut, bien entendu, replacer les possibilités d'action des particuliers dans un cadre plus large. En premier lieu, on rappellera que les juges nationaux sont les juges de droit commun s'agissant de l'application du droit communautaire. On soulignera également la mobilisation croissante des particuliers en direction des procédures non judiciaires qui leur permettent de faire valoir leurs droits : plaintes à la Commission, recours au médiateur, dépôt de pétitions devant le Parlement européen, demandes de documents aux institutions, etc. En outre, les particuliers qui n'ont pu introduire un recours devant la Cour de justice ou qui ont été déboutés peuvent désormais se tourner vers la Cour européenne des droits de l'homme ${ }^{2}$. Toutes ces procédures sont néanmoins d'une efficacité relative et se révèlent, elles aussi, très inégalitaires du point de vue de leur mise en œuvre. Le système de voies d'action et de recours qui permet aux citoyens européens de défendre leurs droits et intérêts est donc complexe et peu lisible ; plus encore, il n'exclut pas certaines incohérences et se montre sujet aux dénis de justice ${ }^{3}$.

Est-ce à dire que les citoyens seraient les oubliés du système juridictionnel de l'Union? S'il est excessif de l'affirmer, il faut remarquer que les juges communautaires, face aux difficultés pratiques qu'ils rencontrent du fait de l'inflation du nombre de recours, envisagent la protection des droits sous une forme plus indirecte, par l'énonciation de standards au niveau européen que les juges nationaux seront tenus de faire respecter. Ils semblent animés par l'idée que leur contribution à la démocratisation de l'Union et à la protection des individus passe davantage par la diffusion de leur jurisprudence et son imposition aux juridictions nationales, que par l'ouverture de leur prétoire. Les juges et référendaires du Tribunal avec lesquels on a pu s'entretenir estimaient ainsi que leur institution a moins vocation à traiter les recours introduits par des citoyens qu'à rendre des jugements exemplaires. Un juge s'est notamment félicité

1. À propos de la remise en cause de la jurisprudence Cassis de Dijon, cf. R. Dehousse, «L'Europe par le droit », art. cité.

2. La Cour européenne des droits de l'homme a récemment admis la recevabilité d'une requête dirigée contre les quinze États-membres de l'Union européenne. Société Guérin Automobiles c. les quinze États-membres, 4 juillet 2000.

3. D. de Bechillon, «Conflits de sentences entre les juges de la loi », Pouvoirs, 96, janvier 2001, p. 107-121. 


\section{Olivier Costa}

de ce que la jurisprudence du Tribunal et de la Cour soit de plus en plus médiatisée et de ce qu'elle concerne avant tout des grandes entreprises, "connues de tous ${ }^{1}$. Il est révélateur que la Cour se soit dotée d'une division de la presse et de la communication, à laquelle elle a attribué des moyens importants. La Cour ne se contente pas de juger : elle fait connaître ses arrêts en les affichant immédiatement, dans toutes les langues officielles de l'Union, sur son site Internet et en accompagnant leur prononcé de communiqués de presse. La Cour multiplie par ailleurs, à l'image de la Commission et du Parlement européen, les initiatives destinées à susciter l'intérêt des citoyens et à les informer de leurs droits. L'organisation de stages pour les juges nationaux à la Cour constitue un autre volet de cette politique. Comme l'affirmait un référendaire du Tribunal : " le droit communautaire n'existe que s'il est connu. C'est un droit original et difficile. Il faut donc le porter à la connaissance des juges et des avocats nationaux, et plus largement des citoyens et des acteurs économiques ${ }^{2}$. Ce travail de médiatisation peut également être compris comme le produit d'une stratégie de légitimation « substantielle » de la Cour, en ce sens qu'il vise à faire connaître au plus grand nombre les « bienfaits » qui s'attachent à ses activités et à sa jurisprudence.

Il est vrai que la jurisprudence de la Cour en matière de protection des droits fondamentaux est considérable. Les juges ont affirmé que ceux-ci faisaient partie intégrante des principes généraux du droit dont ils entendaient assurer le respect ${ }^{3}$. Ils s'inspirent en la matière des traditions constitutionnelles communes aux Étatsmembres, mais aussi des «indications » fournies par les instruments internationaux concernant la protection des droits de l'homme auxquels les États-membres ont coopéré et adhéré, notamment la Convention européenne des droits de l'homme ${ }^{4}$. Depuis l'entrée en vigueur du traité sur l'Union, les juges rappellent ainsi fréquemment que son article $\mathrm{F}$ (article 6 nouveau), paragraphe 2 , fait référence à cette convention ${ }^{5}$. La détermination de ces droits reste toutefois nébuleuse, y compris pour les juristes les plus chevronnés. La proclamation, en décembre 2000 à Nice, de la Charte européenne des droits fondamentaux est un progrès en ce sens, mais la clarification sera limitée compte tenu de la nature purement politique de ce texte.

Il existe donc un fort hiatus entre la pratique actuelle des recours directs devant le Tribunal, qui est délibérément réservée par les juges à une minorité, et les ambitions des représentants des États-membres, qui mettent le droit et l'accès au droit au centre de leurs réflexions sur l'avenir de l'Union, et notamment sur sa légitimation et sa démocratisation. L'affirmation du traité d'Amsterdam selon laquelle 1'Union est « fondée » sur les principes démocratiques commande pourtant une lecture nouvelle de l'architecture institutionnelle de l'Union, de ses objectifs et de son fonctionnement : elle ne peut rester sans effet sur l'accès des citoyens à la protection juridictionnelle. Ils y sont d'ailleurs invités par l'inscription, dans le traité d'Amsterdam égale-

1. Entretien avec un juge du Tribunal, Luxembourg, 17 octobre 2000.

2. Entretien avec un référendaire du Tribunal, Luxembourg, 17 octobre 2000.

3. Sur ce point, cf. E. Bribosia, « La protection des droits fondamentaux dans l'ordre juridique communautaire : le poids respectif des logiques fonctionnelle et autonome dans le cadre normatif et jurisprudentiel », thèse pour le doctorat de droit communautaire, Université libre de Bruxelles, 2000.

4. Arrêts de la Cour du 15 mai 1986, Johnston, C-222/84, Rec. p. 1651, point 18, et du 29 mai 1997, Kremzow, C-299/95, Rec. p. I-2629, point 14.

5. «L'Union respecte les droits fondamentaux, tels qu'ils sont garantis par la Convention européenne de sauvegarde des droits de l'homme et des libertés fondamentales, signée à Rome le 4 novembre 1950 ... ». Cf. l'arrêt du Tribunal, Mayr-Melnhof Kartongesellschaft v. Commission, T-347/94, 14 mai 1998, Rec. p. II-1751. 
ment, d'un principe général de non-discrimination, en raison de l'âge, du sexe, de la race, de l'origine ethnique, de la religion ou des convictions. À plus long terme, la mise en place de l'espace judiciaire annoncé par le traité d'Amsterdam impliquera, aux fins de la lutte contre la «criminalité organisée », l'établissement de certaines incriminations, d'un embryon de droit pénal européen et, en bonne logique, d'organes communautaires de police judiciaire et de justice pénale ${ }^{1}$. Cette évolution remettrait en cause le système judiciaire européen actuel ${ }^{2}$, qui repose sur la collaboration entre juridictions nationales et européenne. Dans une telle hypothèse, la Cour de justice serait appelée à se muer en Cour constitutionnelle ou en Cour suprême. Elle y trouverait certes une confirmation de ses réticences à ouvrir son prétoire aux citoyens, mais ces derniers bénéficieraient (parallèlement à la possibilité d'être directement incriminés par des organes de justice pénale communautaires - si tant est que cela soit un bénéfice) de facultés d'action étendues contre des décisions communautaires auprès des juges nationaux ou de juridictions spécifiques.

Pour l'heure, les possibilités pour les particuliers, et notamment les citoyens, de faire entendre leurs griefs par les juges communautaires et, partant, d'interagir avec le système politique européen, sont très limitées. Le système juridictionnel de l'Union semble perçu par les juges communautaires comme le garant de la bonne application du droit communautaire, au service des objectifs de l'intégration européenne, et non comme un instrument de protection des individus contre les excès des institutions européennes ou comme un vecteur de participation des citoyens à la gouvernance de l'Union. De fait, si l'on peut considérer que l'Union européenne est une Communauté de droit, au sein de laquelle les individus disposent de droits étendus et de garanties quant à leur respect, on ne peut pour autant conclure à son caractère démocratique. En premier lieu, les procédures judiciaires prévues par les traités se révèlent peu susceptibles de fournir aux citoyens les moyens d'un contrôle efficace sur les gouvernants. L'examen de la mise en œuvre de ces procédures donne en effet peu de crédit à l'hypothèse d'une « démocratie judiciaire » qui dépasserait la simple judiciarisation du politique. L'existence de voies de recours ne doit, en second lieu, pas occulter le problème central de la participation et de la perception que les citoyens ont de l'Union européenne. En l'état actuel des choses, la légitimité du droit communautaire et de l'ordre juridique qu'il fonde reste problématique, en raison notamment du sentiment d' «extériorité » que l'Union suscite chez la grande majorité des Européens. À force d'ignorer cette réalité, les acteurs et les zélateurs de la Cour de justice ont développé une conception de l'Union qui s'apparente au Rechtsstaat que défendaient les juristes allemands il y a cent ans ${ }^{3}$, et dont la théorie politique contemporaine a montré les apories et les faiblesses ${ }^{4}$. À l'échelle européenne, plus encore que dans le cadre national, l'existence

1. Cf. G. de Kerchove, A. Weyembergh (dir.), Vers un espace judiciaire pénal européen, Bruxelles, Éditions de l'Université de Bruxelles, 2000.

2. Cf. aussi J.-P. Jacqué, «L'avenir de l'architecture juridictionnelle de l'Union », Revue trimestrielle de droit européen, 1999, p. 443-449.

3. On pense notamment aux écrits de Georg Jellinek (L'État moderne et son droit, Paris, Giard et Brière, 1918, trad. française) et de Hans Kelsen (Théorie pure du droit, Paris, LGDJ, 1999 , trad. française).

4. Pour des réflexions sur l'actualité de la notion, cf. A. Barilari, L'État de droit : réflexion sur les limites du juridisme, Paris, LGDJ, 2000; Michel Troper (dir.), «L'État de droit », Cahiers de philosophie politique et juridique de l'Université de Caen, 24, 1993; D. Colas (dir.), L'État de droit : travaux de la mission sur la modernisation de l'État, Paris, PUF, 1987. Sur le rapport des juges communautaires au positivisme juridique, cf. G. Coron, « La théorie normativiste comme fondement du discours de légitimité de la jurisprudence de la Cour de justice des Communautés européennes », contribution à la journée d'étude du groupe «Europe » de l'AFSP, Paris, 16 mars 2001 (<www.afsp.msh-paris.fr $>$ ). 


\section{Olivier Costa}

d'un État de droit - en l'occurrence d'une Communauté de droit - ne saurait à elle seule épuiser les exigences de la démocratie.

Olivier Costa est chargé de recherche au CNRS (CERVL-Pouvoir, Action publique, Territoire, Institut d'études politiques de Bordeaux). Il a publié « Le Parlement européen entre efficacité fonctionnelle et déficit politique », dans G. Duprat (dir.), L'Union européenne, droit, politique, démocratie, Paris, PUF, 1996 ; «L'Europe par les régions au Parlement de Strasbourg », Critique internationale, 5, 1999 ; «Quelles leçons tirer du manque d'harmonisation des modes de scrutin aux élections européennes?», dans P. Delwit et al. (dir.), Le mode de scrutin fait-il l'élection ?, Bruxelles, Éditions de 1'Université de Bruxelles, 2000. Il a publié récemment Le Parlement européen, assemblée délibérante, Bruxelles, Éditions de 1'Université de Bruxelles, 2001 ; (avec M. Latek) «Paradoxes et limites de la coopération interparlementaire dans l'UE », Revue d'intégration européenne, 23 (2), 2001 ; (avec P. Magnette) « Les transformations de la responsabilité politique dans l'UE », Politiques et Management public, 19 (1), 2001. Ses recherches actuelles portent sur la démocratie et la légitimité dans l'Union européenne, la représentation parlementaire comparée en Europe et les relations entre les responsables politiques infra-étatiques et les acteurs des institutions de 1'Union (CERVL, IEP de Bordeaux, 33607 Pessac Cedex. <o.costa@iep.u-bordeaux.fr>).

\section{RÉSUMÉ/ABSTRACT}

\section{LA COUR DE JUSTICE ET LE CONTRÔLE DÉMOCRATIQUE DE L'UNION EUROPÉENNE}

Face aux difficultés que pose la légitimation du système politique de l'Union européenne, les juristes insistent sur la contribution spécifique de la Cour de justice à ce processus. En reconnaissant aux citoyens de nombreux droits et en leur permettant de les faire sanctionner par divers biais auprès d'elle, la Cour les ferait accéder à un certain degré de contrôle sur les décisions des institutions communautaires. Si l'on se penche sur la jurisprudence et la pratique de la Cour, on constate cependant que les voies de recours sont étroites et qu'il existe de grandes asymétries quant à l'accès des particuliers au prétoire communautaire, que ce soit en fonction de leur nationalité ou de leur degré d'expertise sur les questions européennes. Les juges communautaires semblent d'ailleurs peu pressés de remédier à cette situation. De fait, il est douteux que les possibilités de recours des citoyens devant la Cour contribuent significativement à les rapprocher des acteurs de l'Union et, partant, à améliorer la légitimation de celle-ci. Aussi, la « démocratie judiciaire » à l'échelle supranationale consiste-t-elle pour l'heure en une simple judiciarisation du politique.

\section{THE EUROPEAN COURT OF JUSTICE AND DEMOCRATIC CONTROL OF THE EUROPEAN UNION}

Legal scholars insist on the specific contribution of the European Court of Iustice to the legitimation of the Union's political system. By recognizing many rights to citizens and by making it possible to uphold them by various means, the Court is seen as enabling them to exercise a certain degree of control over the Union institutions'decisions. If one looks at the case-law and the practices of the Court, however, the means of recourse are limited, and there are many disparities in individuals' access to the Union's courtroom, which depends on nationality and the degree of knowledge of European questions. The Union's judges do not seem in a great hurry to remedy this situation. It is dubious that the possibilities of citizens to appeal to the Court will contribute significantly to bringing them closer to the Union's actors, and thereby improve its legitimacy. «Judicial democracy » on the supranational level thus seems to consist of a mere judicialization of politics. 